\title{
Características antropométricas e hábitos alimentares de escolares
}

\author{
Anthropometric features and food habits of students
}

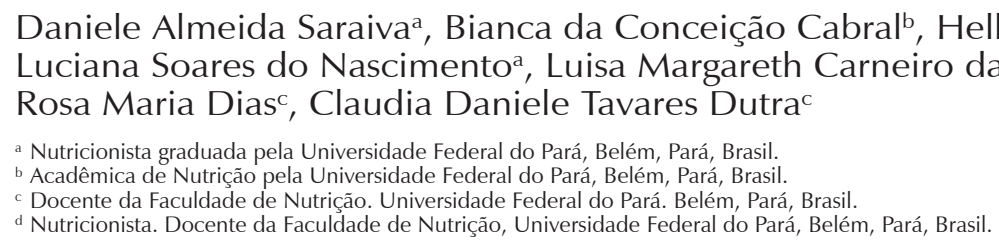

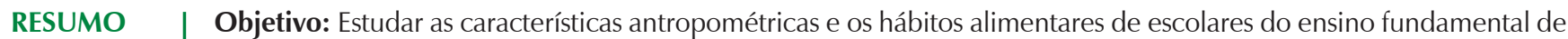
uma escola pública do município de Belém- PA.

Materiais e Métodos: Trata-se de um estudo transversal que analisou dados sociodemográficos, antropométricos e alimentares de escolares do 2 o ao 5ㅇan ano, no período de agosto a dezembro de 2013. Para classificação do estado nutricional foi utilizado o cálculo do índice de massa corporal (IMC), enquanto que a avaliação dos hábitos alimentares foi realizada por meio de um questionário de frequência alimentar.

Resultados: Foram estudados 111 escolares, sendo 51,40\% adolescentes. As crianças e os adolescentes eram na sua maioria do sexo feminino (57,66\%), com média de idade de 8,25 $\pm 0,70$ e 11,01 $\pm 1,60$ anos, respectivamente. Foi observado que a maioria das crianças e adolescentes apresentou estado nutricional adequado. Entretanto foi verificado um percentual considerável de excesso de peso: para as crianças, segundo peso/idade (18,50\%) e IMC/ Idade $(33,30 \%)$ para o sexo feminino $(p=0,016)$, e para os adolescentes (IMC/I: $26,30 \%)$, sem diferença estatística entre os sexos. Os hábitos alimentares indicaram um consumo insuficiente de alimentos considerados protetores para doenças cardiovasculares como: feijão (54,50\%), frutas (70,90\%), salada crua (58,70\%), verduras e legumes cozidos $(58,60 \%)$, e um consumo considerável de alimentos de risco como: carne com gordura (17,9\%), frango com pele $(21,10 \%)$, fritura $(40,20 \%)$, refrigerantes $(41,30 \%)$, salgados fritos e salgadinhos de milho $(36,60 \%)$.

Conclusão: Observou-se que a maioria dos escolares apresentava estado nutricional de eutrofia, entretanto foi constatado um percentual considerável de excesso peso e uma alimentação inadequada.

Palavras-chaves: estado nutricional; hábitos alimentares; escolares.

Objective: To study the anthropometric characteristics and the food habits of basic education students of a public school in the municipality of Belém, Pará.

Materials and Methods: It is a cross-sectional study that analyzed the socio-demographic, anthropometric and dietary data of students from 2nd to 5th year, in the period of August to December 2013. For the nutritional status classification it was utilized the body mass index (BMI), while the evaluation of food habits was performed using a food frequency questionnaire.

Results: One hundred and eleven (111) students were analyzed, $51.40 \%$ teenagers. Children and teenagers were mostly female (57.66\%) with a mean age of $8.25 \pm 0.70$ and $11.01 \pm 1.60$ years, respectively. It was observed that most children and teenagers presented adequate nutritional status. However, it was found a considerable percentage of overweight: for children, according to weight for age $(18.50 \%)$ and BMI for age $(33.30 \%)$ for females $(p=0.016)$, and for teenagers $(B M I / I: 26.30 \%)$, without statistical difference between genders. The food habits indicate an insufficient consumption of foods which are considered protective for cardiovascular diseases, such as: beans (54.50\%), fruits (70.90\%), raw salad (58.70\%), greens and cooked vegetables (58.60\%), and a considerable consumption of risk foods, such as meat with fat (17.9\%), chicken with skin (21.10\%), fried food (40.20\%), soft drinks (41.30\%), fried snacks and corn chips (36.60\%).

Conclusion: It was observed that the majority of the students presented a nutritional status as eutrophic. However, it was found a considerable percentage of overweight and an inadequate nutrition.

Keywords: nutritional status; eating habits; students. 


\section{INTRODUÇÃO}

A transição nutricional tem repercutido na situação de saúde e qualidade de vida das pessoas em diversas partes do mundo, devido às prevalências elevadas de sobrepeso e obesidade que podem desencadear vários danos à saúde da criança ${ }^{1}$, como o desenvolvimento das doenças crônicas não transmissíveis (DCNT).

Atualmente, os países desenvolvidos e subdesenvolvidos definem estratégias para o controle de DCNT, que por sua vez, resultam de uma conduta alimentar não saudável. Este tipo de conduta reflete num padrão de dieta rica em alimentos com alta densidade energética e baixa concentração de nutrientes, de alto consumo de alimentos ultraprocessados, que contém excesso de sódio, gorduras e açúcares. Estes nutrientes apresentam uma relação direta com o aumento de sobrepeso, obesidade, diabetes, hipertensão, dislipidemias e doenças cardiovasculares ${ }^{2}$.

No Brasil, o excesso de peso apresentou um aumento preocupante nas ultimas décadas, não apenas na idade adulta, mas na infância e na adolescência. As crianças e os adolescentes encontram- se com maiores taxas de excesso de peso (33,5\% e 20,5\%, respectivamente) quando comparado com o baixo peso $(4,1 \% \text { e } 3,4 \% \text {, respectivamente) })^{3}$. A avaliação do estado nutricional de escolares constitui-se de grande importância devido às crescentes prevalências de sobrepeso e obesidade em faixas etárias mais jovens, sendo fundamental para identificar os indivíduos em risco, visando à prevenção de agravos nutricionais ${ }^{4}$.

Vale ressaltar que do ponto de vista da Segurança Alimentar e Nutricional, a alimentação saudável, acessível, de qualidade, em quantidade suficiente e de modo permanente é direito de todos. E esta deve ser totalmente baseada em práticas alimentares promotoras da saúde, sem comprometer o acesso a outras necessidades essenciais, além de respeitar as particularidades e características culturais de cada região, cuja valorização e consideração são fundamentais no espaço escolar brasileiro ${ }^{5}$.

Martens et al. ${ }^{6}$ relatam que os hábitos alimentares de crianças sofrem influências das ações educativas realizadas precocemente e que estas devem ser continuas para o sucesso na modificação do comportamento alimentar já adquirido. Segundo Salvi e Ceni ${ }^{7}$ a escola é o ambiente mais propício para o desenvolvimento de estratégias de educação nutricional, devido à relação do trinômio, aluno- famíliacomunidade.

Em face do exposto, o presente trabalho se propõe a estudar as características antropométricas e os hábitos alimentares de escolares do ensino fundamental de uma escola pública do município de Belém-PA.

\section{MATERIAIS E MÉTODOS}

Trata-se de um estudo transversal realizado na escola pública estadual do ensino fundamental Mateus do Carmo do município de Belém- PA, no período de agosto a dezembro de 2013. O estudo abrangeu alunos do $2^{\mathrm{o}}$ ao $5^{\mathrm{o}}$ ano, do turno matutino. O critério de seleção foi baseado no maior número de alunos por turma, sendo selecionada uma turma de cada ano de estudo.

Os pais e/ou responsáveis autorizaram a participação das crianças e adolescentes por meio da assinatura do termo de consentimento livre e esclarecido (TCLE). A participação no estudo foi voluntária, sendo garantido a todos os participantes o abandono do estudo a qualquer momento e o sigilo dos dados e informações apuradas. Não participaram da pesquisa os alunos em que os pais não assinaram o TCLE e que não estiveram presentes no momento da coleta. O estudo foi aprovado pelo Comitê de Ética em Pesquisa do Instituto de Ciências da Saúde da Universidade Federal do Pará, parecer no 392.255/2013.

Para a coleta de dados foi utilizado um protocolo semiestruturado para avaliação dos dados sociodemográficos, características antropométricas e hábitos alimentares. Ressalta-se que os dados sociodemográficos foram respondidos pelos pais ou responsável do aluno. Os dados sociodemográficos estudados foram sexo (masculino e feminino), idade (dividida em faixas etárias de 7 a 9 anos, crianças; e de 10 a 16 anos, adolescentes) $)^{8}$, renda familiar (1 a 2 salários mínimos; 3 a 4 salários mínimos) e cor da pele (branca e não branca), os quais foram divididos de forma arbitrária.

As características antropométricas foram determinadas a partir da aferição de peso e estatura. Sendo o peso aferido em balança digital da marca $\mathrm{SECA}^{\circledR}$ e a estatura em estadiômetro vertical portátil da marca ALTUREXATA ${ }^{\circledR}$, com precisão, respectivamente, de 50 gramas e $1 \mathrm{~mm}$. Para a obtenção do peso, foi solicitado que a criança ficasse descalça e apenas com a vestimenta do uniforme escolar e, na balança, mantivesse os pés completamente apoiados no centro da plataforma, permanecendo ereta e com os braços estendidos e soltos ao longo do corpo. Após a estabilização do marcador, foi registrado o resultado da medida.

Para obtenção da estatura, foi solicitado que a criança se posicionasse no centro do estadiômetro com os pés descalços e a cabeça livre de adereços e mantivesse os pés juntos e os braços estendidos ao longo do corpo. A parte móvel do estadiômetro foi deslizada até a parte superior da cabeça e registrou- se a medida em centímetros ${ }^{9}$. Foi utilizado o cálculo do índice de massa corporal (IMC) para classificação do estado nutricional em baixo peso, adequado, sobrepeso 
e obesidade, sendo as crianças analisadas por meio dos indicadores de peso para idade $(\mathrm{P} / \mathrm{I})$, estatura para idade (E/I) e IMC para idade (IMC/I), enquanto que os adolescentes foram estudados por meio da E/I e IMC/I, conforme as recomendações da Organização Mundial de Saúde ${ }^{10}$. A variável excesso de peso foi constituída pela somatória dos dados de sobrepeso e obesidade.

A avaliação dos hábitos alimentares foi realizada por meio de um questionário de frequência alimentar adaptado (QFA) ${ }^{11}$, semiestruturado, o qual contemplava 11 alimentos, a descrever: carne vermelha, frango, peixe, leite, açaí, frutas, verduras, legumes, feijão, refrigerante, e salgadinhos fritos e industrializados. Para a análise do consumo alimentar foram considerados os dez passos da alimentação saudável para crianças de 2 a 10 anos de idade, conforme as recomendações do Ministério da Saúde ${ }^{12}$.

Os dados obtidos foram armazenados em planilhas eletrônicas do software Microsoft Excel 2000. Para análise utilizou os programas WHO AnthroPlus versão1.0.3, Epi Info versão 3.5.2 e Bioestat versão 5.0, estabeleceu- se como significância valor de $\mathrm{p} \leq 0,05$ e intervalo de confiança de $95 \%$. As variáveis contínuas foram analisadas pelo estudo de medidas de tendência central, como média e mediana, bem como por medidas de variabilidade, como coeficiente de variância e desvio-padrão. As hipóteses foram avaliadas pelos seguintes testes: qui-quadrado com correção de Yates; teste $G$, teste $t$ de student, Exato de Fisher na análise das variáveis categóricas. Os resultados foram apresentados na forma de tabelas e figuras. Os dados qualitativos foram descritos, interpretados e analisados, utilizando-se raciocínios indutivos, dedutivos e comparativos ${ }^{11}$.

\section{RESULTADOS}

Foram estudados 111 escolares, sendo 48,60\% crianças e $51,40 \%$ adolescentes. As crianças e os adolescentes eram na sua maioria do sexo feminino $(57,66 \%)$, com idade

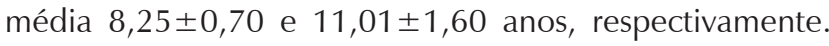
Foi observado que $59,46 \%$ das famílias possuíam a renda familiar de 1 a 2 salários mínimos e que 55,9\% dos escolares possuíam a cor não branca, conforme demonstra a Tabela 1.

$\mathrm{Na}$ análise da avaliação do estado nutricional observouse que a maioria das crianças e adolescente apresentou: E/I adequada (88,90\%; 91,20\%), P/I adequado (81,50\%) e IMC/I adequado $(61,10 \% ; 64,90 \%)$, respectivamente. A magreza foi identificada com um percentual baixo entre as crianças $(5,60 \%)$ e adolescentes (8,80\%). Entretanto foi observado um percentual considerável de excesso de peso para as crianças, segundo Peso/Idade (18,50\%) e IMC/I (33,30\%) para o sexo feminino $(p=0,023)$. Os adolescentes $(26,30 \%)$ apresentaram excesso de peso $(\mathrm{IMC} / \mathrm{I})$, sem diferença estatística entre os sexos (Tabela 2).

Tabela 1 - Distribuição dos escolares segundo as variáveis sociodemográficas.

\begin{tabular}{|c|c|c|c|c|}
\hline \multirow[b]{2}{*}{ Variáveis } & \multirow{2}{*}{$\begin{array}{c}\text { Amostra } \\
\text { n (\%) }\end{array}$} & \multicolumn{2}{|c|}{ Sexo } & \multirow[b]{2}{*}{$p$ valor } \\
\hline & & $\begin{array}{c}\text { Masculino } \\
\text { n (\%) }\end{array}$ & $\begin{array}{c}\text { Feminino } \\
\text { n (\%) }\end{array}$ & \\
\hline Idade (anos) & 111(100) & $47(42,34)$ & $64(57,66)$ & \\
\hline \multicolumn{5}{|l|}{ Faixa etária (anos) } \\
\hline $7-9$ & $54(48,60)$ & $23(42,60)$ & $31(57,40)$ & $0,888^{*}$ \\
\hline $10-16$ & $57(51,40)$ & $24(42,10)$ & $33(57,90)$ & \\
\hline \multicolumn{5}{|l|}{ Escolaridade } \\
\hline $2^{\mathrm{o}}$ ano & $26(23,40)$ & $13(50,00)$ & $13(50,00)$ & $0,406^{* *}$ \\
\hline $3^{\mathrm{o}}$ ano & $26(23,40)$ & $11(42,30)$ & $15(57,70)$ & \\
\hline $4 \mathrm{o}$ ano & $29(26,10)$ & $14(48,30)$ & $15(51,70)$ & \\
\hline $5^{\mathrm{o}}$ ano & $30(27,00)$ & $9(30,00)$ & $21(70,00)$ & \\
\hline \multicolumn{5}{|l|}{ Cor da pele } \\
\hline Branca & $16(14,40)$ & $5(31,25)$ & $11(68,75)$ & $0,215^{* *}$ \\
\hline Não Branca & $62(55,90)$ & $24(38,71)$ & $38(61,29)$ & \\
\hline Não informou & $33(29,70)$ & $18(54,55)$ & $15(45,45)$ & \\
\hline \multicolumn{5}{|l|}{ Renda (salário mínimo) } \\
\hline $1-2$ & $66(59,46)$ & $28(42,42)$ & $38(57,58)$ & $0,241^{* *}$ \\
\hline $3-4$ & $7(6,31)$ & $1(14,29)$ & $6(85,71)$ & \\
\hline Não informou & $38(34,23)$ & $18(47,37)$ & $20(52,63)$ & \\
\hline
\end{tabular}

* Teste Qui-quadrado corrigido de Yates; ** Teste G. 
Na Tabela 3, observou-se que os dados medianos de estatura e de peso das crianças apresentavam-se adequados (WHO), embora, vale ressaltar que tanto a estatura quanto o peso apresentaram valores de até $7,20 \mathrm{~cm}$ e 6,85 kg acima do preconizado para os quartis inferiores $(109,9 \mathrm{~cm} ; 21,3 \mathrm{~kg})$ e superiores $(131,7 \mathrm{~cm} ; 39,4 \mathrm{~kg})$, e um déficit de até $0,10 \mathrm{~cm}$ e $0,70 \mathrm{~kg}$ para os quartis inferiores $(115,0 \mathrm{~cm} ; 17,7 \mathrm{~kg}) \mathrm{e}$ superiores $(138,2 \mathrm{~cm} ; 30,7 \mathrm{~kg})$. As curvas de crescimento de peso das crianças e de IMC para os adolescentes, em comparação às medianas de referência, estão representadas na Figura 1 e evidenciam que os escolares apresentam-se acima da média recomendada pela $\mathrm{WHO}^{10}$.

Tabela 2 - Distribuição de crianças e adolescentes segundo classificação do estado nutricional de estatura para idade, peso para idade e IMC para idade e sexo.

\begin{tabular}{|c|c|c|c|c|c|c|}
\hline \multirow{3}{*}{ Estado nutricional } & \multicolumn{3}{|c|}{ Crianças $n=54(48,60 \%)$} & \multicolumn{3}{|c|}{ Adolescentes $n=57(51,40 \%)$} \\
\hline & \multicolumn{2}{|c|}{ Sexo } & \multirow[b]{2}{*}{$p$ valor } & \multicolumn{2}{|c|}{ Sexo } & \multirow[b]{2}{*}{$p$ valor } \\
\hline & $\begin{array}{c}\text { Masculino } \\
\text { n (\%) }\end{array}$ & $\begin{array}{c}\text { Feminino } \\
\mathrm{n}(\%)\end{array}$ & & $\begin{array}{l}\text { Masculino } \\
\text { n (\%) }\end{array}$ & $\begin{array}{c}\text { Feminino } \\
\mathbf{n}(\%)\end{array}$ & \\
\hline \multicolumn{7}{|l|}{ Estatura/ldade } \\
\hline Estatura baixa & - & $1(100,00)$ & \multirow{3}{*}{$0,292^{*}$} & $1(33,30)$ & $2(66,70)$ & \multirow{3}{*}{$0,304^{*}$} \\
\hline Estatura adequada & $22(45,83)$ & $26(54,17)$ & & $23(44,23)$ & $29(55,77)$ & \\
\hline Estatura elevada & $1(20,00)$ & $4(80,00)$ & & - & $2(100,00)$ & \\
\hline \multicolumn{7}{|l|}{ Peso/Idade } \\
\hline Peso baixo & - & - & \multirow{3}{*}{$0,872 *$} & - & - & \\
\hline Peso adequado & $18(40,91)$ & $26(59,09)$ & & - & - & \\
\hline Peso elevado & $5(50,00)$ & $5(50,00)$ & & - & - & \\
\hline \multicolumn{7}{|l|}{ IMC/Idade } \\
\hline Magreza & $2(66,67)$ & $1(33,33)$ & \multirow{3}{*}{$0,016^{*}$} & $3(60,00)$ & $2(40,00)$ & \multirow{3}{*}{$0,562^{*}$} \\
\hline Adequado & $9(27,27)$ & $24(72,73)$ & & $16(43,24)$ & $21(56,76)$ & \\
\hline Excesso de peso & $12(66,67)$ & $6(33,33)$ & & $5(33,33)$ & $10(66,67)$ & \\
\hline
\end{tabular}

* Teste G.

Tabela 3 - Comparação dos valores medianos de estatura $(\mathrm{cm})$ e peso $(\mathrm{kg})$ de crianças de 7 a 9 anos com o padrão antropométrico da OMS.

\begin{tabular}{|c|c|c|c|c|c|c|c|c|c|c|c|}
\hline \multirow{3}{*}{$\begin{array}{l}\text { Idade } \\
\text { (anos) }\end{array}$} & \multirow{3}{*}{ Sexo } & \multicolumn{5}{|c|}{ Estatura $(\mathrm{cm})$} & \multicolumn{5}{|c|}{ Peso (kg) } \\
\hline & & WHO & Escolar & $\Delta$ & & & WHO & Escolar & $\Delta$ & & \\
\hline & & $\mathbf{a}$ & B & b-a & Z & $\boldsymbol{p}^{*}$ & $\mathbf{a}$ & B & b-a & $\angle$ & $p^{*}$ \\
\hline \multirow{2}{*}{7} & M & 121,70 & 126,00 & 4,30 & 0,81 & \multirow{6}{*}{0,386} & 22,90 & 22,20 & $-0,70$ & $-0,22$ & \multirow{6}{*}{0,469} \\
\hline & $\mathrm{F}$ & 120,80 & 128,00 & 7,20 & 1,31 & & 22,40 & 22,90 & 0,50 & 0,15 & \\
\hline \multirow{2}{*}{8} & M & 127,30 & 130,70 & 3,45 & 0,62 & & 25,40 & 26,30 & 0,90 & 0,24 & \\
\hline & $\mathrm{F}$ & 126,60 & 126,50 & $-0,10$ & $-0,01$ & & 25,00 & 24,70 & $-0,30$ & $-0,09$ & \\
\hline \multirow{2}{*}{9} & M & 132,60 & 136,70 & 4,15 & 0,41 & & 28,10 & 34,95 & 6,85 & 1,12 & \\
\hline & $\mathrm{F}$ & 132,50 & 131,70 & $-0,75$ & $-0,33$ & & 28,20 & 29,35 & 1,15 & 0,00 & \\
\hline
\end{tabular}

* Teste $t$ de Student; $\Delta$ : valores medianos observados menos valores medianos de referência; WHO: valores medianos da Organização Mundial de Saúde;

Z: pontos de corte de classificação de estatura e peso para idade.

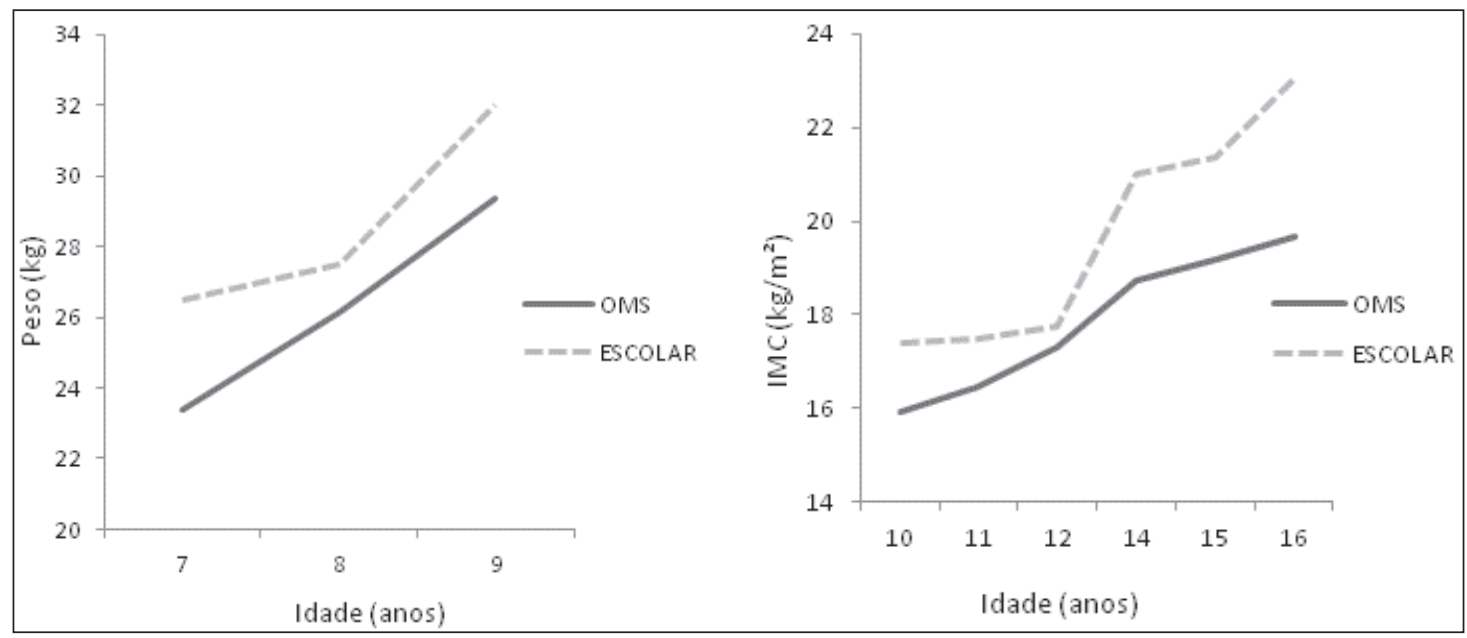

Figura 1. Curva de crescimento de peso de crianças e IMC de adolescentes em comparação com o padrão antropométrico da OMS. 
Tabela 4 - Frequência absoluta e relativa do consumo de alimentos dos escolares, segundo o sexo.

\begin{tabular}{|c|c|c|c|c|}
\hline \multirow[b]{2}{*}{ Consumo de alimentos } & \multirow{2}{*}{$\begin{array}{c}\text { Amostra } \\
\text { n (\%) }\end{array}$} & \multicolumn{2}{|c|}{ Sexo } & \multirow[b]{2}{*}{$p$ valor } \\
\hline & & $\begin{array}{c}\text { Masculino } \\
\text { n (\%) }\end{array}$ & $\underset{\mathrm{n}(\%)}{\text { Feminino }}$ & \\
\hline \multicolumn{5}{|l|}{ Carne vermelha com gordura } \\
\hline Sim & $15(17,90)$ & $9(60,00)$ & $6(40,00)$ & $0,326^{* *}$ \\
\hline Não & $69(82,10)$ & $29(42,00)$ & $40(58,00)$ & \\
\hline \multicolumn{5}{|l|}{ Frango com pele } \\
\hline $\operatorname{Sim}$ & $23(21,10)$ & $13(56,50)$ & $10(43,50)$ & $0,151^{* *}$ \\
\hline Não & $86(78,90)$ & $32(37,20)$ & $54(62,80)$ & \\
\hline \multicolumn{5}{|l|}{ Peixe frito } \\
\hline $\operatorname{Sim}$ & $41(40,20)$ & $19(46,30)$ & $22(53,70)$ & $0,506^{* *}$ \\
\hline Não & $61(59,80)$ & $23(37,70)$ & $38(62,30)$ & \\
\hline \multicolumn{5}{|l|}{ Leite } \\
\hline $\operatorname{Sim}$ & $88(70,30)$ & $34(38,60)$ & $54(61,40)$ & $0,190^{* *}$ \\
\hline Não & $23(20,70)$ & $13(56,50)$ & $10(43,50)$ & \\
\hline \multicolumn{5}{|l|}{ Açaí } \\
\hline Sim & $106(95,50)$ & $44(41,50)$ & $62(58,50)$ & $0,356^{*}$ \\
\hline Não & $5(4,50)$ & $3(60,00)$ & $2(40,00)$ & \\
\hline \multicolumn{5}{|l|}{ Frutas } \\
\hline $\operatorname{Sim}(7 x$ semana $)$ & $25(29,10)$ & $10(40,00)$ & $15(60,00)$ & $0,986^{* *}$ \\
\hline Não (<7x semana) & $61(70,90)$ & $26(42,60)$ & $35(57,40)$ & \\
\hline \multicolumn{5}{|l|}{ Salada crua } \\
\hline $\operatorname{Sim}(7 x$ semana $)$ & $26(41,30)$ & $9(34,60)$ & $17(65,40)$ & $0,821^{* *}$ \\
\hline Não (<7x semana) & $37(58,70)$ & $13(32,10)$ & $24(64,90)$ & \\
\hline \multicolumn{5}{|l|}{ Verduras e legumes cozidos } \\
\hline $\operatorname{Sim}(7 x$ semana $)$ & $24(41,40)$ & $8(33,10)$ & $16(66,70)$ & $0,438^{* *}$ \\
\hline Não (<7x semana) & $34(58,60)$ & $16(47,10)$ & $18(52,90)$ & \\
\hline \multicolumn{5}{|l|}{ Feijão } \\
\hline $\operatorname{Sim}(7 x$ semana $)$ & $50(45,50)$ & $24(48,00)$ & $26(52,00)$ & $0,314^{* *}$ \\
\hline Não (<7x semana) & $60(54,50)$ & $22(36,70)$ & $38(63,30)$ & \\
\hline \multicolumn{5}{|l|}{ Refrigerante } \\
\hline $\operatorname{Sim}(\leq 2 x$ semana $)$ & $54(58,70)$ & $24(44,40)$ & $30(55,60)$ & $0,948^{* *}$ \\
\hline Não (>2x semana) & $38(41,30)$ & $18(47,40)$ & $20(52,60)$ & \\
\hline \multicolumn{5}{|l|}{ Salgados fritos e salgadinhos de milho } \\
\hline $\operatorname{Sim}(\leq 2 x$ semana $)$ & $59(63,40)$ & $25(42,40)$ & $34(57,60)$ & $0,957^{* *}$ \\
\hline Não (>2x semana) & $34(36,60)$ & $15(44,10)$ & $19(55,90)$ & \\
\hline
\end{tabular}

* Teste Exato de Fisher; ** Teste Qui-quadrado corrigido de Yates.

A avaliação do consumo alimentar demonstrada na Tabela 4 indicou um consumo insuficiente de alguns alimentos, segundo as recomendações do Ministério da Saúde ${ }^{12}$, como: frutas $(70,90 \%)$, salada crua $(58,70 \%)$, verduras e legumes cozidos (58,60\%) e feijão (54,50\%); e um consumo considerável de carne com gordura $(17,9 \%)$, frango com pele $(21,10 \%)$, peixe frito $(40,20 \%)$, refrigerantes $(41,30 \%)$ e salgados fritos e salgadinhos de milho $(36,60 \%)$. Porém, não houve diferença estatística significativa entre os sexos. Foi observado que a maioria dos escolares apresentava o hábito de consumir leite $(70,30 \%)$ e açaí $(95,50 \%)$.

\section{DISCUSSÃO}

O excesso de peso e os hábitos alimentares em escolares são considerados sérios problemas da atualidade por constituírem fatores de riscos para as doenças cardiovasculares, dislipidemias, diabetes, hipertensão arterial e cânceres em todas as idades ${ }^{13}$. Dessa forma, a escola apresenta- se como um ambiente estratégico no incentivo à formação de hábitos alimentares saudáveis por meio do desenvolvimento de ações preventivas aos desvios nutricionais, em razão dos comportamentos, atitudes e hábitos serem bem estruturados nesta fase ${ }^{6}$. 
A análise dos dados sociodemográficos demonstrados nesta pesquisa, observou que a maioria dos participantes pertencia ao sexo feminino e eram adolescentes, apresentando a cor da pele "não branca" e uma renda familiar em torno de 1 a 2 salários mínimos. Esses dados são semelhantes ao estudo realizado por Mariz et al. ${ }^{14}$, no interior da Paraíba com 109 escolares.

A característica de indivíduos "não brancos" deve-se a miscigenação histórica presente no Brasil, não havendo como definir precisamente a cor devido à mistura de raças ${ }^{15}$. Estudo relata que a renda está diretamente ligada ao poder de compra do indivíduo, onde crianças e adolescentes oriundas de famílias com renda alta têm maior prevalência de apresentar sobrepeso e obesidade comparada àquelas com renda baixa ${ }^{16}$. No entanto, com a transição nutricional, já é observada a prevalência de excesso de peso nas classes menos favorecidas, visto tal tendência no presente trabalho, que estudou escolares de baixa renda.

A classificação do estado nutricional demonstrou neste estudo, que a condição de adequação foi maior tanto em crianças quanto em adolescentes, porém, notou-se que aproximadamente um terço das crianças e adolescentes apresentou excesso de peso, dados que também foram encontrados nos estudos de Pedrosa et al. ${ }^{17}$ e Medeiros et al. ${ }^{18}$ com crianças, na prevalência de $29,91 \%$ e $23,50 \%$, respectivamente. Souza e Enes ${ }^{19}$, em sua pesquisa com adolescentes observaram um percentual ainda maior de excesso de peso. Estes resultados são preocupantes, uma vez que o excesso de peso é considerado grande problema de saúde pública por acarretar múltiplas doenças, principalmente em faixas etárias cada vez mais jovens ${ }^{20}$.

Com relação ao sexo, a prevalência de excesso de peso foi maior no sexo masculino para as crianças, e para os adolescentes houve uma maior frequência para o sexo feminino, sem diferença estatística, estando de acordo com a pesquisa de Krinski et al. ${ }^{21}$, que encontraram os mesmos resultados para os adolescentes de uma rede pública de ensino da cidade de Vilhena em Rondônia. Enquanto, que o IBGE $^{3}$ apontou que o excesso de peso em crianças aumentou entre indivíduos do sexo masculino, de 10,9\% em 1974 para $34,8 \%$ em 2009; e no sexo feminino, de $8,6 \%$ para $32 \%$.

Estudos têm demonstrado que as taxas de excesso de peso e obesidade estão aumentando em todas as faixas etárias, enquanto que a desnutrição está diminuindo, comprovando a transição nutricional presente no Brasil e no mundo ${ }^{22}$. Dados estes que estão de acordo com este trabalho, onde se verificou a prevalência de excesso de peso nos escolares.

Com relação aos dados medianos de altura e peso das crianças avaliadas, verificou-se que as crianças apresentavam a altura acima em todas as faixas etárias, em ambos os sexos, com déficit, apenas, para as idades de 08 e 09 anos do sexo feminino, em comparação aos dados medianos da OMS. Os dados medianos do peso também apresentaram na sua maioria acima dos valores de referência, principalmente na faixa etária de 09 anos de idade do sexo masculino. Entretanto, observou- se déficit de peso, nas idades de 07 e 08 anos, do sexo masculino e feminino, respectivamente. Com isso, é fundamental uma alimentação adequada nessa faixa etária, considerando a evolução de altura e de peso medianos ligeiramente superiores aos valores de referência ${ }^{10}$.

A curva de crescimento demonstrou que todas as crianças analisadas encontraram-se acima da média do padrão antropométrico, supondo que estas se alimentam adequadamente ou apresentam uma dieta rica em alimentos energéticos (gorduras e açúcares) que provavelmente está associada ao excesso de peso corporal. Já a curva de crescimento de IMC de adolescentes também mostrou que os adolescentes estão superiores à média de referência da OMS. Vale ressaltar, que o aumento do IMC pode aumentar o número de pessoas com DCNTs, que consequentemente eleva as taxas de mortalidade ${ }^{23}$.

Os dados da análise do consumo alimentar revelaram que os escolares não tinham hábito de consumir alimentos benéficos para saúde (protetores) como frutas, verduras e legumes e feijão em concordância com a pesquisa do IBGE ${ }^{24}$ e Santos et al. ${ }^{16}$, que verificaram o consumo de frutas $31,5 \%$ e $34,0 \%$, e hortaliças $31,2 \%$ e $44,0 \%$, respectivamente, com diferenças apenas para o feijão, onde $62,6 \%$ e $76,0 \%$ consumiam feijão, regularmente. De acordo com Spinelli ${ }^{4}$, o maior consumo de hortaliças está associado à diminuição do IMC.

Foi observado que os alimentos de risco para DCNT como carne com gordura, frango com pele, fritura, refrigerantes e salgadinhos de milho fazem parte do hábito alimentar da comunidade escolar, semelhantes aos dados do IBGE $^{24}$ e Carvalho et al..$^{25}$. Para Meireles ${ }^{26}$, o consumo de carne com gordura $(44,0 \%)$ e frango com pele $(34,9 \%)$ foi maior em comparação a este trabalho que encontrou $17,90 \%$ e $21,10 \%$, respectivamente. Sendo assim, faz- se necessário evitar estes tipos de alimentos, pelo fato do elevado consumo desses contribuírem para o aumento da prevalência de excesso de peso e suas comorbidades ${ }^{14}$.

Ressalta-se que os alimentos mais consumidos pelos escolares foram o leite e o açaí. Não obstante, os resultados encontrados por Spinelli et al. ${ }^{4}$ e Santos et al. ${ }^{16}$ verificaram que $52 \%$ e $49 \%$, respectivamente tinham o hábito de consumir leite. Tal alimento é importante para o crescimento e desenvolvimento de crianças, além de favorecer a ingestão 
de cálcio. Supõe-se que o elevado consumo de açaí devese ao hábito alimentar da região. Esta fruta apresenta alto valor calórico e nutricional, rica em ácidos graxos essenciais, fibras, vitaminas e minerais como cálcio, potássio e ferro (biodisponibilidade relativa), além da grande quantidade de antioxidantes ${ }^{27}$.

O processo de modificação dos hábitos alimentares é bastante complexo, principalmente devido ao padrão alimentar atual e a necessidade da elaboração de estratégias de educação nutricional junto ao indivíduo, a fim de que o mesmo seja capaz de compreender a necessidade e a importância do problema ocasionado pelos maus hábitos alimentares, que exercem grande influência no desenvolvimento das DCNT ${ }^{14}$.

Os resultados deste estudo mostraram que a maioria dos escolares apresentava estado nutricional de eutrofia. Entretanto, foi observado um percentual considerável de excesso peso e uma alimentação inadequada, caracterizada pela baixa ingestão de feijão, frutas, verduras e legumes e alto consumo de carne com gordura, frango com pele, fritura, refrigerantes e salgadinhos de milho. Foi observado, ainda um elevado consumo de açaí e leite pela comunidade escolar. Diante desse contexto, a escola, juntamente com uma equipe multiprofissional, apresenta papel fundamental na promoção de hábitos alimentares saudáveis para o bom crescimento e desenvolvimento dos escolares, e na prevenção de riscos futuros para o acometimento das doenças crônicas não transmissíveis.

\section{REFERÊNCIAS}

1. Kaufmann CC, Albernaz EP. Prevalência e fatores associados ao excesso de peso em crianças de uma coorte no Sul do Brasil. Cienc. Saude 2013;6(3):172-80.

2. Brasil. Ministério da Saúde. Secretaria de Atenção à Saúde. Departamento de Atenção Básica. Política Nacional de Alimentação e Nutrição. Brasília (DF): Ministério da Saúde; 2013. 84 p.

3. Brasil. Ministério da Saúde. Instituto Brasileiro de Geografia e Estatística - IBGE. Ministério do Planejamento, Orçamento e Gestão. Pesquisa de Orçamentos Familiares 2008-2009: Antropometria e Estado nutricional de Crianças, Adolescentes e Adultos no Brasil. Rio de Janeiro: IBGE; 2010. 130 p.

4. Spinelli MGN, Morimoto JM, Freitas APG, Barros CM, Dias DHS, Pioltine MB, Gonçalves PPO, Navarro RB. Estado nutricional e consumo alimentar de pré- escolares e escolares de escola privada. Cienc Saude 2013;6(2):94-101.

5. Aquilla R. A educação alimentar e nutricional no espaço escolar: saber, sabor e saúde [dissertação]. Ijuí (RS): Universidade Regional do Noroeste do Estado do Rio Grande do Sul, 2011.

6. Silva EB, Martens IBG, Tuma RB, Siqueira YM. Educação nutricional aplicada à mudança de conhecimentos, atitudes e práticas alimentares dos escolares de uma Escola de Belém- PA. Nutr pauta 2012;112:41-5.
7. Salvi C, Ceni GC. Educação nutricional para pré-escolares da Associação Creche Madre Alix. Vivências Rev Eletronica Extensão URI 2009;5(8):71-6.

8. Brasil. Ministério da Saúde. Secretaria de Atenção à Saúde. Departamento de Ações Programáticas e Estratégicas. Brasília (DF): Ministério da Saúde; 2010.

9. Brasil. Ministério da Saúde. Secretaria de Atenção à Saúde. Departamento de Atenção Básica. Orientações para a coleta e análise de dados antropométricos em serviços de saúde: norma técnica do Sistema de Vigilância Alimentar e Nutricional - SISVAN. Brasília (DF): Ministério da Saúde; 2011. 76 p.

10. World Health Organization. WHO Reference 2007: growth reference data 5-19 years. [online]. Switzerland: WHO; 2007. [capturado 2014 Jan 02]. Disponível em: http://www.who.int/ growthref/en.

11. Fisberg RM, Martini LA, Slater B. Métodos de inquéritos alimentares. In: Fisberg RM, Slater B, Marchioni DML, Martini LA. Inquéritos alimentares: métodos e bases científicos. São Paulo: Manole; 2005. p. 1-31.

12. Brasil. Ministério da Saúde. Secretaria de Atenção à Saúde. Coordenação Geral da Política de Alimentação e Nutrição. Dez passos para uma alimentação saudável para crianças de 2 a 10 anos. Brasília (DF): Ministério da Saúde; 2007.

13. Alcântara Neto OD, Silva RCR, Assis AMO, Pinto EJ. Fatores associados à dislipidemia em crianças e adolescentes de escolas públicas de Salvador, Bahia. Rev Bras Epidemiol 2012;15(2): 335-45. http://dx.doi.org/10.1590/S1415-790X2012000200011

14. Mariz LS, Medeiros CCM, Vieira CENK, Enders BC, Coura AS. Modificação na frequência alimentar de crianças e adolescentes: acompanhamento em serviço de referência. Rev Latino-Am Enfermagem 2013;21(4):973-81. http://dx.doi.org/10.1590/S010411692013000400020

15. Ribeiro AA. Sobre uma "pedagogia da morenidade": gênero e mestiçagem entre estudantes de duas escolas de Belém do Pará. Rev acad educa do ISE Vera Cruz 2012;2(1):114-29. http://dx.doi. org/10.14212/veras.vol2.n1.ano2012.art82

16. Santos GG, Sousa JB, Toscano MB, Morais MEA. Hábitos alimentares e estado nutricional de adolescentes de um centro de juventude da cidade de Anápolis. Ens cienc: Ciências biológicas, agrárias e da saúde 2011;15(1):141-51.

17. Pedrosa OP, Neto WNR, Silva AC, Pinho ST, Alves DM. Composição corporal de escolares da rede de ensino pública e privada em uma cidade da Região Norte. Anais da Semana Educa 2010; 1(1).

18. Medeiros CCM, Cardoso MAA, Pereira RAR, Alves GTA, França ISX, Coura AS, Carvalho DF. Estado nutricional e hábitos de vida em escolares. Rev Bras Crescimento Desenvolvimento Hum 2011;21(3):789-97.

19. Souza JB, Enes CC. Influência do consumo alimentar sobre o estado nutricional de adolescentes de Sorocaba-SP. J Health Sci Inst 2013;31(1):65-70.

20. Brasil. Ministério da Saúde. Secretária de Atenção à Saúde. Departamento de Atenção Básica. Saúde da criança: nutrição infantil - aleitamento materno e alimentação complementar. Brasília (DF): Ministério da Saúde, 2009. 112 p. 
21. Krinski K, Elsangedy HM, Hora S, Rech CR, Legnani E, Santos BV, Campos W, Silva SG. Estado nutricional e associação do excesso de peso com gênero e idade de crianças e adolescentes. Rev bras cineantropom desempenho hum 2011;13(1):29-35.

22. De Paula DV, Botelho LP, Zanirati VF, Lopes ACS, Santos LC. Avaliação nutricional e padrão de consumo alimentar entre crianças beneficiárias e não beneficiárias de programas de transferência de renda, em escola municipal do Município de Belo Horizonte, Estado de Minas Gerais, Brasil, em 2009. Epidemiol Serv Saúde 2012;21(3):385-94. http://dx.doi.org/10.5123/S167949742012000300004

23. Brasil. Ministério da Saúde. Secretária de Vigilância em Saúde. Departamento de Análise de Situação de Saúde. Plano de ações estratégicas para o enfretamento das doenças crônicas não transmissíveis (DCNT) no Brasil 2011-2022. Brasília (DF): Ministério da Saúde, 2011. 148 p.
24. Brasil. Ministério do Planejamento, Orçamento e Gestão. Instituto Brasileiro de Geografia e Estatística-IBGE. Diretoria de Pesquisas. Coordenação de População e Indicadores Sociais. Pesquisa Nacional de Saúde do Escolar 2009. Rio de Janeiro: IBGE; 2009.

25. Carvalho AP, Oliveira VB, Santos LS. Hábitos alimentares e práticas de educação nutricional: atenção a crianças de uma escola municipal de Belo Horizonte, Minas Gerais. Pediatria (São Paulo) 2010;32(1):20-7.

26. Meireles AL. Xavier CC, Sales ADF, Abreu MNS, Malta DC, Caiaffa WT. Perfil alimentar de adolescentes e adultos jovens em Belo Horizonte segundo perfil antropométrico e contexto de moradia. Rev Med Minas Gerais 2013;23(supl 2):S12-9.

27. Portinho JA, Zimmermann LM, Bruck MR. Efeitos benéficos do Açaí. Int J Nutr 2012;5(1):15-20. 